\title{
Bifurcation Analysis of Zellner's Marshallian Macroeconomic Model
}

\author{
Sanjibani Banerjee ${ }^{\mathrm{a}}$, William A Barnett ${ }^{\mathrm{b}, *}$, \\ Evgeniya A Duzhak ${ }^{c}$, and Ramu Gopalan ${ }^{\mathrm{d}}$ \\ ${ }^{a}$ University of Kansas, Lawrence, $K S$ \\ ${ }^{\mathrm{b}}$ University of Kansas, Lawrence, $K S$ \\ ${ }^{\mathrm{c}}$ Baruch College, CUNY, New York, NY \\ ${ }^{\mathrm{d}}$ Washington and Jefferson College, Washington, $P A$
}

\begin{abstract}
The Marshallian Macroeconomic Model in Zellner and Israilevich (2005) provides a novel way to examine sectoral dynamics through the introduction of a dynamic entry/exit equation in addition to the usual demand and supply functions found in models of this class. In this paper we examine the possibility of cyclical behavior in the Marshallian Macroeconomic Model and investigate the existence of a Hopf bifurcation with respect to the parameter in the entry/exit equation.
\end{abstract}

Key words: Marshallian Macroeconomic Model, Dynamic Entry/Exit, Hopf Bifurcation, Limit Cycles

JEL: C61, C62, E32, L16

* Corresponding author: William A Barnett

Email address: barnett@ku.edu 


\section{Introduction}

With the formulation of the Marshallian Macroeconomic Model (MMM) by Veloce and Zellner (1985) for a single sector, and later extension to a multi-sector model by Zellner and Israilevich (2005), Zellner and his co-authors attempted to incorporate sectoral dynamics and its effects on aggregates and vice-versa. One of the novel features of this model was explicitly to formulate the dynamics of firm entry and exit within industries. With this in mind the basic MMM is described by sectoral demand, supply, and entry/exit equations. In the later version of the model in Zellner and Israilevich (2005), factor markets, the government, and a monetary sector were added to complete the model.

The entry/exit behavior modeled in the MMM can be described by the equation $\frac{\dot{N}}{N}=\gamma^{\prime}\left(\Pi-F^{e}\right)$, i.e. the growth rate of firms in the industry is proportional to the difference in current industry profitability, $\Pi$, and the long-run future profitability in the industry, $F^{e}$. The speed of adjustment is determined by the parameter, $\gamma^{\prime}$. Zellner and Israilevich (2005) describe the emergence of rich dynamics in key variables, such as price and output at the sectoral, as well as at the aggregate, level once an entry/exit equation for each industry is introduced into the model . In the simulation exercises conducted by Zellner and Israilevich (2005), $\gamma^{\prime}$ and $F^{e}$ were fixed parameters. Varying these parameters would change the equilibria and could possibly cause changes in the nature of the equilibria, such as the number of solutions and the stability properties of the equilibria. In this paper, we undertake this task of examining the model's characteristics with respect to the entry/exit parameter $F^{e}$ by searching for a bifurcation within the theoretically feasible parameter space.

Examining the existence of bifurcations in dynamic economic models has important consequences from a theoretical as well as an empirical perspective. Grandmont (1985) showed that it was possible for even the most classical dynamic, general equilibrium, macroeconomic models to demonstrate stable solutions or more complex solutions in the form of cycles or chaos. The reason behind such disparate behavior was not a difference in the structure of the model, but the fact that the parameter space of such models was stratified into subsets or bifurcation regions, each of which supported a very different kind of dynamics. As Barnett (2000) pointed out, it is possible for economists having different policy views to agree on structurally similar or identical models, but with the parameters being in different bifurcation subsets of the parameter space. This conclusion is in contrast with the earlier view that different policy views must imply different structural models.

Bifurcation analysis of parameter space stratification is a fundamental and frequently overlooked approach to exploring model dynamic properties. Basic properties of any dynamic system are stability and the nature of its disequilibrium dynamics. Just as it is important to know for what parameter values a system is stable or unstable, it is equally important to know the nature of stability ( e.g. monotonic convergence, damped single periodic convergence, or damped multi-periodic convergence) or instability (periodic, multi-periodic, or chaotic). Informally we say that a system has 
undergone a bifurcation if a small, smooth change in a parameter value(s) produces a sudden topological change in the nature of singular points and trajectories of the system.

When the values of a system's parameters are not known with certainty, bifurcation analysis can provide meaningful insights into solution dynamics of the system. If a confidence region around a parameter estimate includes a bifurcation point, then various kinds of dynamics can be consistent with the parameter being within the confidence interval. In such cases the robustness of inferences about dynamics is damaged.

Bifurcations can be local or global. Local bifurcations are examined through linearization of a non-linear system around its equilibrium, since in general non-linear systems tend to behave in the same manner as the linear system in a close neighborhood of the equilibrium. At a bifurcation point, the number of equilibrium may change. There may also be changes in the stability properties of equilibrium points and/or changes in the nature of orbits near the equilibrium. Examples of local bifurcations include saddle-node bifurcation, transcritical bifurcation, pitch-fork bifurcation, period-doubling(flip) bifurcation, and Hopf bifurcation.

Examining the existence of bifurcation has important consequences for theoretical and empirical model building in economics. Boldrin and Woodford (1990) have given an extensive survey of developments in dynamic, general equilibrium theory and conditions under which endogenous fluctuations are possible. Benhabib and Nishimura (1979) show that the optimal growth path becomes a closed orbit in a multi-sector model for some discount rate values within the theoretically feasible region. Benhabib and Day (1982) and Grandmont (1985) have also shown the possibility of chaotic behavior in general equilibrium models.

More recent work on detecting bifurcations in macroeconomic models have been undertaken by Barnett and his co-authors. Barnett and He (2002) show the existence of a transcritical and Hopf bifurcation for different policy parameters in the dynamic, continuous time macroeconometric model of Bergstrom et al. (1992). Furthermore, Barnett and He $(2004,2006,2010)$ find the existence of a singularity induced bifurcation within the empirical parameter space of the Leeper and Sims (1994) Euler equations model for the US economy. Barnett and Duzhak (2008, 2010) recently found the presence of period-doubling and Hopf bifurcation in New Keynesian models.

Section 2, describes the MMM and the derivations of the dynamic equations governing the path of output in each sector. In Section 3 we discuss the possibility of cyclical behavior in MMM and present our result of a Hopf bifurcation. Finally, Section 4 concludes the paper and indicates some future extensions. 


\section{The Model}

We consider a two sector, continuous time version of the Marshallian Macroeconomic Model (MMM), as outlined in Zellner and Israilevich (2005). Each sector is characterized by an aggregate demand function for its output, an aggregate supply function, and aggregate input demand functions for labor and capital. We also include the government that collects taxes on output, purchases output from the two sectors, and inputs from the factor markets. Although Zellner and Israilevich (2005) include money markets in their original model with monetary services being used as a factor of production, we exclude the presence of money markets from the model at this stage to make our analysis simpler.

\subsection{Output Demand}

Since both the government and households demand goods from the two sectors, the total demand for goods in the $i$ th sector, $i=1,2$, is sum of the demands from the government and the aggregate demand from households. As in Zellner and Israilevich (2005), these demands are given exogenously, but some of the other factors ${ }^{1}$ determining household demand are omitted for simplicity. Aggregate demand is thus given by

$$
S_{i}=G_{i}+P_{i}^{1-\eta_{i i}} P_{j}^{\eta_{i j}}\left(S\left(1-T^{s}\right)\right)^{\eta_{i s}},
$$

where $G_{i}$ is the nominal government expenditure in sector $i, S=S_{1}+S_{2}$ is the total income (nominal ouput), $T^{s}$ is the tax rate, $\eta_{i i}$ is the own price elasticity, $\eta_{i j}$ is the cross price elasticity, and $\eta_{i s}$ is the income elasticity. Expressed in terms of growth rates, the aggregate demand for goods in each sector is the weighted sum of growth rates $^{2}$ of demand from the government and households

$$
\hat{S}_{i}=g_{i} \hat{G}_{i}+\left(1-g_{i}\right)\left[\left(1-\eta_{i i}\right) \hat{P}_{i}+\eta_{i j} \hat{P}_{j}+\eta_{i s}\left(\hat{S}+\hat{T}^{s^{\prime}}\right)\right]
$$

where $g_{i}$ is the ratio of government spending in sector $i$ to total sales in sector $i$ and $T^{s^{\prime}}=1-T^{s}$.

\subsection{Output Supply}

There are $N_{i}$ identical firms in the $i$ th sector, each using a Cobb-Douglas type production function, $q_{i}=A_{i}^{*} L_{i}^{\alpha} K_{i}^{\beta}$, with $0<\alpha_{i}, \beta_{i}<1$ and $0<\theta_{i}=1-\alpha_{i}-\beta_{i}<1$, where $A_{i}^{*}$ is the product of a neutral technological change, and labor and capital augmentation factors, and is assumed in this paper to be a constant.

1 The other demand shift variables that Zellner and Israilevich (2005) indicate include the number of households, money balances, and demand trends.

2 Given any variable $X \in \mathbb{R}$, we denote $\dot{X}=\frac{d X}{d t}$ and $\hat{X}=\frac{\dot{X}}{X}$. 
The aggregate nominal profit-maximizing output supply of each sector, $i$, is the number of firms in the sector, $N_{i}$, times the nominal profit-maximizing supply of each firm within that sector. This is given by $S_{i}=N_{i} P_{i}^{\frac{1}{\theta_{i}}} w^{\frac{-\alpha_{i}}{\theta_{i}}} r^{\frac{-\beta_{i}}{\theta_{i}}}$, where $P_{i}, w$, and $r$ are the price, wage rate, and rental rate respectively. Expressing this in growth rates, we have

$$
\hat{S}_{i}=\hat{N}_{i}+\frac{1}{\theta_{i}} \hat{P}_{i}-\frac{\alpha_{i}}{\theta_{i}} \hat{w}-\frac{\beta_{i}}{\theta_{i}} \hat{r}
$$

\subsection{Entry/Exit}

We consider the simplest form of the entry/exit equation proposed by Zellner and Israilevich (2005),

$$
\hat{N}_{i}=\gamma_{i}\left[\Pi_{i}-F_{i}\right],
$$

where $\Pi_{i}=\theta_{i} S_{i}$ is the current nominal aggregate industry profit for sector $i$, while $F_{i}$ represents the aggregate long-run equilibrium profits in sector $i$, taking account of discounted entry costs. The parameters $F_{i}$ and $\gamma_{i}$ are both positive and could be functions of time. In our analysis, as in the work of Zellner and Israilevich (2005), we will consider them to be time invariant. The coefficient, $\gamma_{i}$, is the speed of adjustment for sector $i$. Given that $\gamma_{i}$ is assumed to be positive, we can interpret the entry/exit equations as follows. A positive departure from equilibrium profits, $F_{i}^{e}$, will attract new firms into the industry, while a negative departure will induce firms to leave the industry. The larger the value of $\gamma_{i}$, the faster will be this adjustment.

\subsection{Government}

Total nominal, government expenditure, $G$, is the sum of expenditures in each of the two sectors, $G_{i}$, and its expenditures on labor, $G_{L}$, and capital, $G_{K}$. Zellner and Israilevich (2005) assume that each component of government expenditure, $G_{i}$, for all $i=1,2, L, K$, grows at the same rate as the total government expenditure. We accommodate this assumption by proposing that $G_{i}=\zeta_{i} G$, where $\zeta_{i}$ is the fraction of total government expenditure in the $i$ th market. Thus in terms of growth rates, we have $\hat{G}_{i}=\hat{G}$.

The government collects a single uniform tax at the rate $T^{s}$ on output (in this paper we omit corporate taxes for simplicity). The tax revenue, $R$, is thus given by $R=T^{s} \cdot S$, which expressed in terms of growth rates yields $\hat{R}=\hat{T}^{s}+\hat{S}$.

Zellner and Israilevich (2005) further assume that there is an exogenously determined deficit/surplus, $D$, defined as the government expenditures as a percentage of revenues, so that $D=\frac{G}{R}$. Thus the flow budget identity of the government in terms of growth rates is

$$
\hat{G}=\hat{D}+\hat{R}=\hat{D}+\hat{T}^{s}+\hat{S}
$$




\subsection{Factor Markets}

Given the Cobb-Douglas technologies, the aggregate, profit-maximizing factor demands from sector $i$ are $L_{i}=\frac{\alpha_{i} S_{i}}{w}$ and $K_{i}=\frac{\beta_{i} S_{i}}{r}$. The government demand for labor and capital are $L_{g}=\frac{G_{L}}{w}$ and $K_{g}=\frac{G_{K}}{r}$, respectively. The total demand for each factor in terms of growth rates is then the weighted sum of growth rates of sectoral demands and the government demand for that factor, as stated below in 2.6 and 2.7.

$$
\begin{gathered}
\frac{L_{1}}{L} \hat{L_{1}}+\frac{L_{2}}{L} \hat{L_{2}}+\frac{L_{g}}{L} \hat{L_{g}}=l_{1}{\hat{L_{1}}}+l_{2}{\hat{L_{2}}}+l_{g}{\hat{L_{g}}}, \\
\frac{K_{1}}{K} \hat{K}_{1}+\frac{K_{2}}{K} \hat{K}_{2}+\frac{K_{g}}{K} \hat{K}=k_{1} \hat{K}_{1}+k_{2} \hat{K}_{2}+k_{g} \hat{K}_{g} .
\end{gathered}
$$

The explicit dependence of the weights, $l_{i}$ and $k_{i}$, on $S_{1}$ and $S_{2}$ is given in Appendix A.

The supply of factors of production is again assumed to be exogenous, as in Zellner and Israilevich (2005), $L=\left(\frac{w}{P}\right)^{\delta}\left(\frac{S}{P}\right)^{\delta_{s}}$ and $K=\left(\frac{r}{P}\right)^{\phi}\left(\frac{S}{P}\right)^{\phi_{s}}$, where $\delta$ (resp. $\phi$ ) and $\delta_{s}\left(\right.$ resp. $\left.\phi_{s}\right)$ are price and income elasticities of labor (resp. capital). Here again we leave out the 'other factors' for simplicity. In terms of growth rates, the labor and capital supplies equal

$$
\begin{aligned}
& \hat{L}=\delta(\hat{w}-\hat{P})+\delta_{s}(\hat{S}-\hat{P}), \\
& \hat{K}=\phi(\hat{r}-\hat{P})+\phi_{s}(\hat{S}-\hat{P}) .
\end{aligned}
$$

\subsection{Quantity and Price Aggregates}

The growth rate of aggregate nominal sales and the price aggregate are given by

$$
\begin{aligned}
& \hat{S}=s_{1} \hat{S}_{1}+s_{2} \hat{S}_{2}, \\
& \hat{P}=s_{1} \hat{P}_{1}+s_{2} \hat{P}_{2},
\end{aligned}
$$

where $s_{i}=\frac{S_{i}}{S}$.

\subsection{Solving the model}

The above model is solved using market clearing conditions in all markets, that is output and factor markets, and incorporating the government's flow budget identity. The complete solution procedure is outlined in Appendix A. We are able to reduce all these equations to yield the following two dynamic equations, which govern the behavior of $S_{1}$ and $S_{2}$ :

$$
\left[\begin{array}{c}
\dot{S}_{1} \\
\dot{S}_{2}
\end{array}\right]=\left[\begin{array}{c}
\mathcal{F}_{1}\left(S_{1}, S_{2} ; \boldsymbol{\Omega}\right) \\
\mathcal{F}_{2}\left(S_{1}, S_{2} ; \Omega\right)
\end{array}\right]=\mathcal{F}\left(S_{1}, S_{2} ; \boldsymbol{\Omega}\right) .
$$


The explicit form of the non-linear functions $\mathcal{F}_{1}$ and $\mathcal{F}_{2}$ can be found in Appendix A. The vector $\Omega$ consists of all structural parameters. The assumed values for these parameters are given in Appendix B. We consider $F_{1}$, the entry parameter for sector 1 , as our bifurcation parameter, when we look for a co-dimension 1 bifurcation in the following section.

An equilibrium for this model would constitute a value of $\left(S_{1}, S_{2}\right)$, at which $\dot{S}_{1}=0$ and $\dot{S}_{2}=0$, i.e. in the System $2.12, \mathcal{F}\left(S_{1}, S_{2} ; \Omega\right)=0$. As given in Appendix A:

$$
\mathcal{F}\left(S_{1}, S_{2} ; \boldsymbol{\Omega}\right)=\left(\mathcal{H}\left(S_{1}, S_{2} ; \boldsymbol{\Omega}\right)\right)^{(-1)} \mathcal{D}\left(S_{1}, S_{2} ; \boldsymbol{\Omega}\right),
$$

where $\mathcal{H}$ is a matrix of dimension $2 \times 2$ and $\mathcal{D}$ is a vector of dimension $2 \times 1$. The elements of $\mathcal{H}$ and $\mathcal{D}$ are an indication of the high degree of nonlinearity involved in determining the dynamics of the above mentioned equilibrium. As expected, there will be several equilibria, which can arise due to this non-linearity in $\mathcal{F}$.

However, it is easy to see from Equation 2.13 that the values of $S_{1}$ and $S_{2}$, at which $\mathcal{D}=0$, will always be an equilibrium. Under the assumption that there is no growth in government deficit $(D)$ and taxes $\left(T^{s}\right)$, this solution directly corresponds to the solution of the entry/exit equation given in Equation 2.4, so that

$$
S_{1}=\frac{1}{\theta_{1}} F_{1} \quad \text { and } \quad S_{2}=\frac{1}{\theta_{2}} F_{2} .
$$

These solutions are economically relevant, since they are positive and ensure that there is no further entry/exit in both sectors, implying a long run equilibrium. In the next section, we examine stability and perform a bifurcation analysis of this equilibrium.

\section{Stability and Bifurcation Analysis of Equilibrium}

The dynamics in this two sector MMM, in terms of convergence to the equilibrium given 2.14, can be described by generalizing the analysis of the one sector MMM by Veloce and Zellner (1985). Using the entry/exit equation assumed in Zellner and Israilevich (2005) and solving the one sector model as in Veloce and Zellner (1985) would yield the following differential equation, which is equivalent to 2.12 for the one sector model:

$$
\dot{S}=a S(S-F)
$$

where $a$ depends on the structural parameters. Here the stationary solution, $S=F$, is stable, if and only if $a<0$, which is true, if and only if demand is inelastic. This result can be understood by the following argument, adapted from Veloce and Zellner (1985). Suppose $S>F$, so that current profitability is greater than equilibrium profitability. Then firms will enter, causing the market supply to increase, resulting in a lower price. This drop in price will result in a lower aggregate sales, $S$, (because of the inelasticity of demand), decreasing the difference between the current profitability, $S$, and equilibrium profitability, $F$. With the one sector dynamics governed 
by the logistic function given above, this process will result in a monotonic path to equilibrium in a continuous time model. On the other hand, if demand is elastic, then the solution is unstable and any deviation from the equilibrium will result in divergence.

However, in a multisector model, we need to consider the effects of cross price and income elasticities along with own price elasticity. Two interesting features arise with respect to the equilibrium dynamics in the multisector model. First, unlike the one sector model, even when the two sectors have elastic demand (own price elasticity greater than 1), the solution may be stable. This is true for the calibrated parameter values generating Figure 1. Second, the path to the long run equilibrium may not be monotonic, so it may depict oscillatory behavior. This is also true for the calibrated parameters generating Figures 1 and 2.

To understand why these results may obtain, suppose the two sectors produce normal goods that are substitutes and have elastic demand. Also suppose Sector 1 is out of equilibrium (say, $\left.S_{1}>\frac{1}{\theta_{1}} F_{1}\right)^{3}$, while Sector 2 is at equilibrium (i.e. $\left.S_{2}=\frac{1}{\theta_{2}} F_{2}\right)^{4}$. Since current profitability is higher than equilibrium profitability, entry takes place in Sector 1, which increases industry supply and, in turn, causes a drop in Sector 1 price, $P_{1}$. This decline in $P_{1}$ will affect industry sales through two channels. One, since demand is elastic the decline in price will cause industry sales, $S_{1}$, to increase. Two, the decline in $P_{1}$ will cause a decrease in Sector 2 demand (the goods being substitutes), causing a decline in Sector 2 price, $P_{2}$, and quantity, $Q_{2}$, and hence a decline in Sector 2 sales, $S_{2}$. If this decline in $S_{2}$ is greater in magnitude than the initial increase in $S_{1}$, then aggregate sales (or nominal income), $S=S_{1}+S_{2}$, will decline. Since the goods are normal goods, this decline in $S$ will result in a fall in $S_{1}$. So even though the former effect, which increases $S_{1}$, tends to be destabilizing, the cross price and aggregate income effect may offset, in turn, this potentially destabilizing influence.

It is thus clear that there are two opposing effects on $S_{1}$, when Sector 1 profitability is greater than equilibrium profitability. If the second, negative effect dominates the first, positive effect, then $S_{1}$ will decrease, bringing it closer to its equilibrium level, $\frac{1}{\theta_{1}} F_{1}$. This would imply that, even though there are more firms in Sector 1 , they are each producing less, given the cross price and income effects stemming from changes in Sector 2. Also notice that the decline in $P_{1}$ has caused a decrease in Sector 2 demand and hence a decline in $S_{2}$, pushing Sector 2 sales below the equilibrium level, $\frac{1}{\theta_{2}} F_{2}$. We can adapt the previous analysis and and apply it to the case of Sector 2 sales being below the equilibrium level, which could ultimately result in $S_{2}$ increasing. This increase in $S_{2}$ will again, through the income effect, result in a rise in $S_{1}$. With these opposing effects, the interaction between the magnitudes of the shifts and elasticities could cause a cyclical convergence. See Figure 1 for an example.

3 This argument can be easily adapted to the case of Sector 2 .

4 Note that this analysis will be valid, even if $S_{2}$ is not initially at the equilibrium level. 
The above explanation indicates an oscillatory convergence to equilibrium. It is worth mentioning that this delicate mechanism depends crucially on the own price, cross price, and income elasticities, and the magnitude of shifts in demand and supply in each sector. It is definitely possible that these shifts are not sufficient and may result in the solution being unstable.

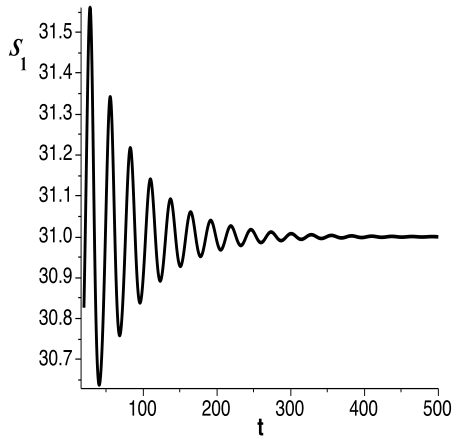

(a)

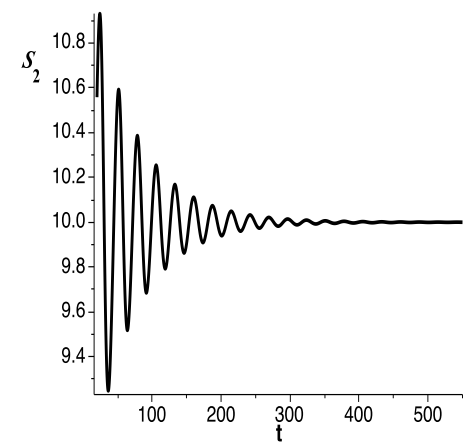

(b)

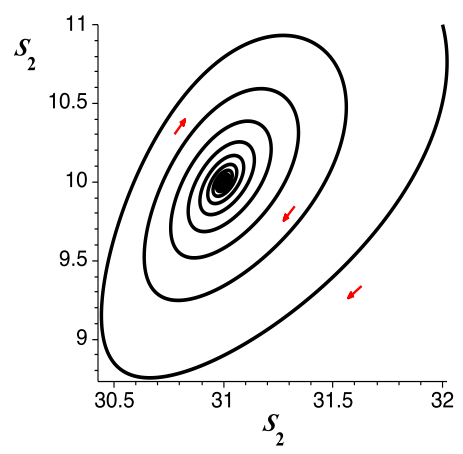

(c)

Figure 1. Oscillatory convergence for $F_{1}=6.2>F^{H}$.

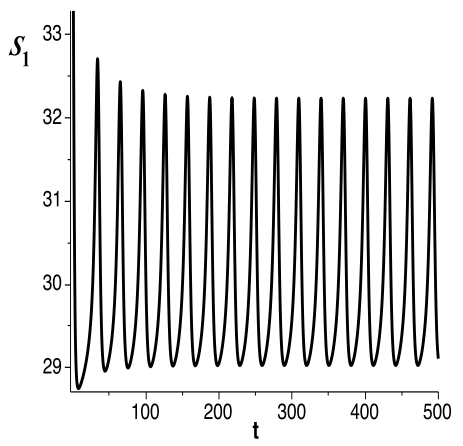

(a)

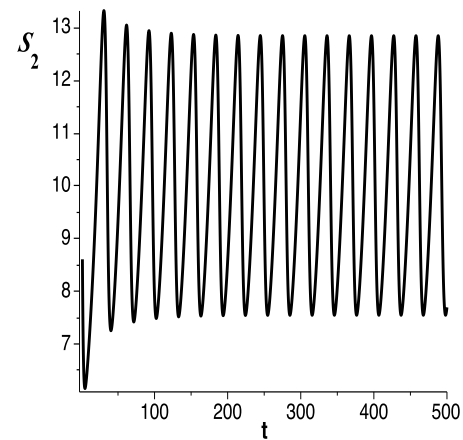

(b)

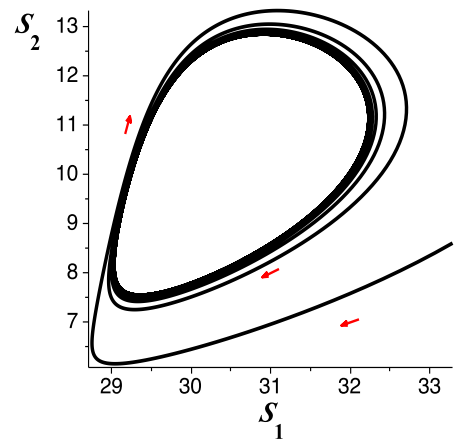

(c)

Figure 2. Stable cycles for $F_{1}=6<F^{H}$.

We emphasize that, for an oscillatory convergence, the elasticity parameters need to be consistent with values of the other parameters in production, input markets, entry/exit equations, and government policy. If some of these parameters were to change, then it is very likely that the economy may go from cyclical convergence to persistent cycles or even explosive behavior. Figures 1 and 2 depict a change in the dynamics from oscillatory convergence to stable cycles, when the entry/exit parameter is changed in one sector. In order to investigate this possibility in the MMM, we look for a bifurcation within the theoretically feasible parameter space.

We now examine the existence of a Hopf bifurcation of codimension-1. The codimension, as defined by Kuznetsov (2004), is the number of independent conditions determining the bifurcation boundary. In the following analysis, we vary only parameter $F_{1}$, while all other parameters will be maintained at their theoretically feasible values given in Appendix B. This procedure of varying a single parameter helps us to identify a codimension-1 bifurcation. In particular, we will investigate the presence of a Hopf (or Andronov-Hopf) bifurcation, which occurs when the the Jacobian of 
$\mathcal{F}$ has a pair of purely imaginary eigenvalues at some critical value of the parameter $F_{1}$.

In order to analyze a codim-1 Hopf bifurcation for the System 2.12, we first look for the value of $\left(S_{1}, S_{2}\right)$ and the bifurcation parameter $\left(F_{1}\right)$ at which the following conditions hold simultaneously:

$$
\begin{aligned}
\mathcal{F}_{1}\left(S_{1}, S_{2}, F_{1}\right) & =0, \\
\mathcal{F}_{2}\left(S_{1}, S_{2}, F_{1}\right) & =0, \\
\operatorname{tr}\left(\mathbf{J}_{\mathcal{F}}\left(S_{1}, S_{2}, F_{1}\right)\right) & =0, \\
\operatorname{det}\left(\mathbf{J}_{\mathcal{F}}\left(S_{1}, S_{2}, F_{1}\right)\right) & >0,
\end{aligned}
$$

where $\mathbf{J}_{\mathcal{F}}$ is the Jacobian of $\mathcal{F}$. Equations 3.1 and 3.2 yield the equilibrium for the system of differential equations in 2.12. In particular, we pay attention to the solution given in 2.14. Conditions 3.3 and 3.4 are sufficient to ensure that the eigenvalues of $\mathbf{J}_{\mathcal{F}}$ are purely imaginary. Figure 3 gives the plots of the trace and determinant of $\mathbf{J}_{\mathcal{F}}$ as functions of $F_{1}$. It is clear that at the computed critical value, $F^{H}=6.070386762$, Conditions 3.3 and 3.4 are satisfied and the slope of the trace is not zero ${ }^{5}$, implying a Hopf bifurcation. Thus as the parameter $F_{1}$ crosses $F^{H}$ from the right, the solution given in 2.14 goes from a stable equilibrium to an unstable one. In fact, the system is locally spiralling inward for $F_{1}>F^{H}$, and for $F_{1}$ close enough $^{6}$ to $F^{H}$ and $F_{1}<F^{H}$, the system exhibits stable cycles ${ }^{7}$ in the phase space. See Figures 1 and 2 .

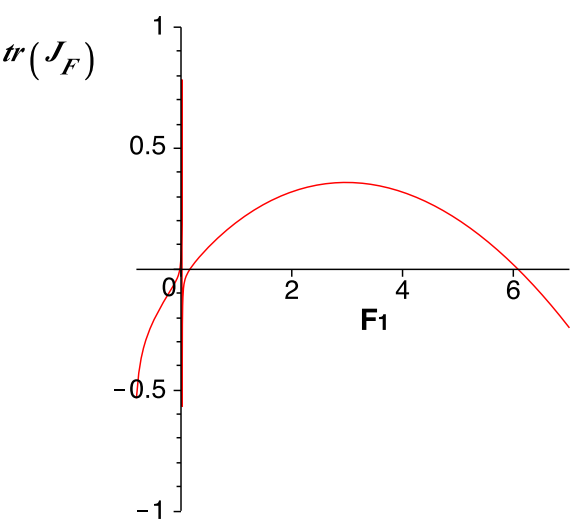

(a) Trace of $J_{F}$

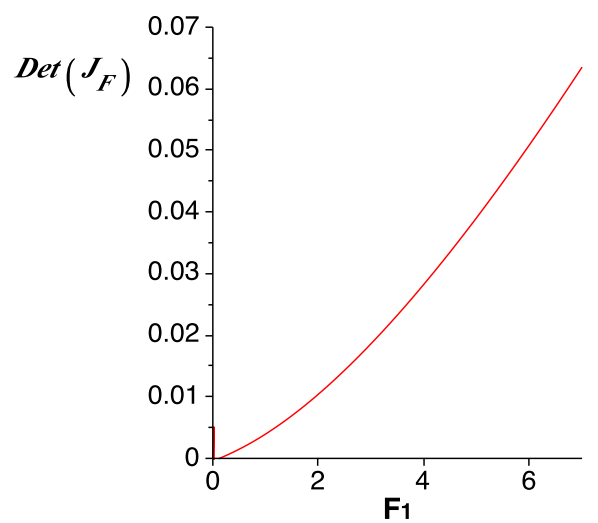

(b) Determinant of $J_{F}$

Figure 3. Trace and Determinant of $\mathbf{J}_{\mathcal{F}}$

5 The computed slope of the trace was $\left.\frac{d\left(\operatorname{tr}\left(\mathbf{J}_{\mathcal{F}}\left(F_{1}\right)\right)\right)}{d F_{1}}\right|_{F_{1}=F^{H}}=0.16265 \neq 0$, thus satisfying the transversality condition in Kuznetsov (2004).

6 Periodic orbits arise for $5.89743<F_{1}<F^{H}$.

7 For the limit cycles to be stable, the first Lyapunov coefficient $\ell_{1}$ needs to be negative. In this case $\ell_{1}=-5171543705$, thus satisfying the non-degeneracy condition in Kuznetsov (2004). 


\section{Conclusions and Extensions}

In this paper we study a special, nested case of the two sector MMM and investigated the possibility of cyclical behavior, which could arise due to certain combinations of own price, cross price, and income elasticities. We also showed that a Hopf bifurcation exists within the theoretically feasible parameter space, giving rise to stable cycles. Our choice of $F_{1}$ as the candidate for bifurcation parameter re-emphasizes the importance of a dynamic entry/exit equation in models of this class. There are several avenues that need to be explored in future work. One such possibility would be the introduction of expectations in firms' future profitability. As a future research agenda, we plan to introduce the money market and examine the possibility of other kinds of bifurcations with respect to government and monetary policy parameters. 


\section{Appendix A. Deriving the Dynamic Equations.}

Firstly, we recall that the government's flow budget identity in growth rates is

$$
\hat{G}=\hat{R}+\hat{D}=\hat{D}+\hat{T}^{s}+s_{1} \hat{S}_{1}+s_{2} \hat{S}_{2}
$$

Using the assumption that each component of the government expenditure grows at the same rate as total government expenditure, the output demand functions in terms of growth rates can be expressed as:

$$
\hat{S}_{i}=g_{i} \hat{G}+\left(1-g_{i}\right)\left[\left(1-\eta_{i i}\right) \hat{P}_{i}+\eta_{i j} \hat{P}_{j}+\eta_{i s}\left(\hat{S}+\hat{T}^{s^{\prime}}\right)\right]
$$

In this expression, we also relabeled taxes by $T^{s^{\prime}}=\left(1-T^{s}\right)$ and thus $\hat{T}^{s^{\prime}}=\frac{-T^{s}}{\left(1-T^{s}\right)} \hat{T}^{s}$. The weight, $g_{i}$, can be shown to explicitly depend on the level variables $S_{1}$ and $S_{2}$ in order to yield

$$
g_{i}=\frac{G_{i}}{S_{i}}=\frac{\zeta_{i} D T^{s}\left(S_{1}+S_{2}\right)}{S_{1}} .
$$

We can now express the system of demand equations given by Equation A.2 in matrices and solve for growth rate of prices as follows.

$$
\mathcal{N}\left[\begin{array}{l}
\hat{P}_{1} \\
\hat{P}_{2}
\end{array}\right]=\mathcal{A}\left[\begin{array}{l}
\hat{S}_{1} \\
\hat{S}_{2}
\end{array}\right]+\left[\begin{array}{l}
C_{1} \\
C_{2}
\end{array}\right]
$$

where,

$$
\begin{gathered}
\mathcal{N}=\left[\begin{array}{cc}
\left(1-\eta_{11}\right) & \eta_{12} \\
\eta_{21} & \left(1-\eta_{22}\right)
\end{array}\right], \\
\mathcal{A}=\left[\begin{array}{cc}
\frac{1}{1-g_{1}}+\left[\left(1-\eta_{1 s}\right)-\frac{1}{1-g_{1}}\right] s_{1} & \left(1-\eta_{1 s}-\frac{1}{1-g_{1}}\right) s_{2} \\
\left(1-\eta_{2 s}-\frac{1}{1-g_{2}}\right) s_{1} & \frac{1}{1-g_{2}}+\left[\left(1-\eta_{2 s}\right)-\frac{1}{1-g_{2}}\right] s_{2}
\end{array}\right],
\end{gathered}
$$

and

$$
\left[\begin{array}{l}
C_{1} \\
C_{2}
\end{array}\right]=\left[\begin{array}{l}
\left(1+\eta_{1 s} \frac{T^{s}}{1-T^{s}}-\frac{1}{1-g_{1}}\right) \hat{T}^{s}-\frac{g_{1}}{1-g_{1}} \hat{D} \\
\left(1+\eta_{2 s} \frac{T^{s}}{1-T^{s}}-\frac{1}{1-g_{2}}\right) \hat{T}^{s}-\frac{g_{1}}{1-g_{1}} \hat{D}
\end{array}\right]
$$

Assumption 1. We assume that the cross price elasticities are such that $\mathcal{N}$ is invertible.

We denote $\mathcal{P}=\mathcal{N}^{-1}$ and $\mathcal{B}=\mathcal{P} \mathcal{A}$ to obtain the following:

$$
\left[\begin{array}{l}
\hat{P}_{1} \\
\hat{P}_{2}
\end{array}\right]=\mathcal{P} \mathcal{A}\left[\begin{array}{l}
\hat{S}_{1} \\
\hat{S}_{2}
\end{array}\right]+\mathcal{P}\left[\begin{array}{l}
C_{1} \\
C_{2}
\end{array}\right]=\mathcal{B}\left[\begin{array}{l}
\hat{S}_{1} \\
\hat{S}_{2}
\end{array}\right]+\mathcal{P}\left[\begin{array}{l}
C_{1} \\
C_{2}
\end{array}\right]
$$


Moving to the factor markets, the growth rate of aggregate profit maximizing labor demand from each sector, $i$, is $\hat{L}_{i}=\hat{S}_{i}-\hat{w}$, and the labor demand from the government is $\hat{L}_{g}=\hat{G}-\hat{w}$. Since the total demand for labor is the sum of sectoral demand and the government demand for labor, the growth rate of the total demand for labor is given by the following weighted sum

$$
\frac{L_{1}}{L} \hat{L_{1}}+\frac{L_{2}}{L} \hat{L_{2}}+\frac{L_{g}}{L} \hat{L_{g}}=l_{1} \hat{L_{1}}+l_{2} \hat{L_{2}}+l_{g} \hat{L_{g}}
$$

where the weights are determined by the level variables as follows:

$$
\begin{aligned}
& l_{i}=\frac{L_{i}}{L}=\frac{\alpha_{i} S_{i}}{\alpha_{1} S_{1}+\alpha_{2} S_{2}+\zeta_{l} D T^{s}\left(S_{1}+S_{2}\right)}, \\
& l_{g}=\frac{L_{g}}{L}=\frac{\zeta_{l} D T^{s}\left(S_{1}+S_{2}\right)}{\alpha_{1} S_{1}+\alpha_{2} S_{2}+\zeta_{l} D T^{s}\left(S_{1}+S_{2}\right)} .
\end{aligned}
$$

Equating the growth rates of labor demand and labor supply, we can solve for growth rate of equilibrium wage rate

$$
\begin{array}{r}
\hat{w}=\frac{1}{1+\delta}\left\{\left(l_{1}+\left(l_{g}-\delta_{s}\right) s_{1}\right) \hat{S}_{1}+\left(l_{2}+\left(l_{g}-\delta_{s}\right) s_{2}\right) \hat{S}_{2}\right. \\
\left.\left.+\left(\delta+\delta_{s}\right)\left(s_{1} \hat{P}_{1}+s_{2} \hat{P}_{2}\right)+l_{g}\left(\hat{D}+\hat{T}^{s}\right)\right)\right\}
\end{array}
$$

Manipulating the capital market equilibrium conditions similar to the labor market equilibrium conditions, we calculate the growth rate of aggregate capital demand as

$$
\hat{K}=\frac{K_{1}}{K} \hat{K}_{1}+\frac{K_{2}}{K} \hat{K}_{2}+\frac{K_{g}}{K} \hat{K}=k_{1} \hat{K}_{1}+k_{2} \hat{K}_{2}+k_{g} \hat{K}_{g}
$$

where the weights are determined by the level variables as follows:

$$
\begin{aligned}
& k_{i}=\frac{K_{i}}{K}=\frac{\beta_{i} S_{i}}{\beta_{1} S_{1}+\beta_{2} S_{2}+\zeta_{k} D T^{s}\left(S_{1}+S_{2}\right)}, \\
& k_{g}=\frac{K_{g}}{K}=\frac{\zeta_{k} D T^{s}\left(S_{1}+S_{2}\right)}{\beta_{1} S_{1}+\beta_{2} S_{2}+\zeta_{k} D T^{s}\left(S_{1}+S_{2}\right)} .
\end{aligned}
$$

Equating the growth rates of capital demand and capital supply, we can solve for growth rate of equilibrium rental rate

$$
\begin{gathered}
\hat{r}=\frac{1}{1+\phi}\left\{\left(k_{1}+\left(k_{g}-\phi_{s}\right) s_{1}\right) \hat{S}_{1}+\left(k_{2}+\left(k_{g}-\phi_{s}\right) s_{2}\right) \hat{S}_{2}\right. \\
\left.+\left(\phi+\phi_{s}\right)\left(s_{1} \hat{P}_{1}+s_{2} \hat{P}_{2}\right)+k_{g}\left(\hat{D}+\hat{T}^{s}\right)\right\} .
\end{gathered}
$$

Now we can substitute $\hat{N}_{i}, \hat{w}, \hat{r}$, and $\hat{P}_{i}$ into the output supply (growth rate) equations to find $\dot{S}_{1}$ and $\dot{S}_{2}$. Let us define 


$$
\begin{aligned}
& \mathcal{H}=\left\{\left[\begin{array}{ll}
\theta_{1} & 0 \\
0 & \theta_{2}
\end{array}\right]\right. \\
& -\left[\begin{array}{cc}
\frac{\left(S_{1}+S_{2}\right)-\left(\frac{\alpha_{1}\left(\delta+\delta_{s}\right)}{(1+\delta)}+\frac{\beta_{1}\left(\phi+\phi_{s}\right)}{(1+\phi)}\right) S_{1}}{\left(S_{1}+S_{2}\right)} & \frac{-\left(\frac{\alpha_{1}\left(\delta+\delta_{s}\right)}{(1+\delta)}+\frac{\beta_{1}\left(\phi+\phi_{s}\right)}{(1+\phi)}\right) S_{2}}{\left(S_{1}+S_{2}\right)} \\
\frac{-\left(\frac{\alpha_{2}\left(\delta+\delta_{s}\right)}{(1+\delta)}+\frac{\beta_{2}\left(\phi+\phi_{s}\right)}{(1+\phi)}\right) S_{1}}{\left(S_{1}+S_{2}\right)} & \frac{\left(S_{1}+S_{2}\right)-\left(\frac{\alpha_{2}\left(\delta+\delta_{s}\right)}{(1+\delta)}+\frac{\beta_{2}\left(\phi+\phi_{s}\right)}{(1+\phi)}\right) S_{2}}{\left(S_{1}+S_{2}\right)}
\end{array}\right]\left[\begin{array}{ll}
B_{11} & B_{12} \\
B_{21} & B_{22}
\end{array}\right] \\
& +\left[\begin{array}{ll}
\frac{\alpha_{1}\left(l_{1}\left(S_{1}+S_{2}\right)+\left(l_{g}-\delta_{s}\right) S_{1}\right)}{(1+\delta)\left(S_{1}+S_{2}\right)} & \frac{\alpha_{1}\left(l_{2}\left(S_{1}+S_{2}\right)+\left(l_{g}-\delta_{s}\right) S_{2}\right)}{(1+\delta)\left(S_{1}+S_{2}\right)} \\
\frac{\alpha_{2}\left(l_{1}\left(S_{1}+S_{2}\right)+\left(l_{g}-\delta_{s}\right) S_{1}\right)}{(1+\delta)\left(S_{1}+S_{2}\right)} & \frac{\alpha_{2}\left(l_{2}\left(S_{1}+S_{2}\right)+\left(l_{g}-\delta_{s}\right) S_{2}\right)}{(1+\delta)\left(S_{1}+S_{2}\right)}
\end{array}\right] \\
& \left.+\left[\begin{array}{cc}
\frac{\beta_{1}\left(k_{1}\left(S_{1}+S_{2}\right)+\left(k_{g}-\phi_{s}\right) S_{1}\right)}{(1+\phi)\left(S_{1}+S_{2}\right)} & \frac{\beta_{1}\left(k_{2}\left(S_{1}+S_{2}\right)+\left(k_{g}-\phi_{s}\right) S_{2}\right)}{(1+\phi)\left(S_{1}+S_{2}\right)} \\
\frac{\beta_{2}\left(k_{1}\left(S_{1}+S_{2}\right)+\left(k_{g}-\phi_{s}\right) S_{1}\right)}{(1+\phi)\left(S_{1}+S_{2}\right)} & \frac{\beta_{2}\left(k_{2}\left(S_{1}+S_{2}\right)+\left(k_{g}-\phi_{s}\right) S_{2}\right)}{(1+\phi)\left(S_{1}+S_{2}\right)}
\end{array}\right]\right\}\left[\begin{array}{cc}
\frac{1}{S_{1}} & 0 \\
0 & \frac{1}{S_{2}}
\end{array}\right], \\
& \mathcal{D}=\left[\begin{array}{l}
\theta_{1} \gamma_{1}\left(\theta_{1} S_{1}-F_{1}\right) \\
\theta_{2} \gamma_{2}\left(\theta_{2} S_{2}-F_{2}\right)
\end{array}\right] \\
& -\left[\begin{array}{c}
\frac{\alpha_{1} l_{g}\left(\hat{D}+\hat{T}^{s}\right)}{1+\delta}+\frac{\beta_{1} k_{g}\left(\hat{D}+\hat{T}^{s}\right)}{1+\phi} \\
\frac{\alpha_{2} l_{g}\left(\hat{D}+\hat{T}^{s}\right)}{1+\delta}+\frac{\beta_{2} k_{g}\left(\hat{D}+\hat{T}^{s}\right)}{1+\phi}
\end{array}\right] \\
& +\left[\begin{array}{cc}
\frac{\left(S_{1}+S_{2}\right)-\left(\frac{\alpha_{1}\left(\delta+\delta_{s}\right)}{(1+\delta)}+\frac{\beta_{1}\left(\phi+\phi_{s}\right)}{(1+\phi)}\right) S_{1}}{\left(S_{1}+S_{2}\right)} & \frac{-\left(\frac{\alpha_{1}\left(\delta+\delta_{s}\right)}{(1+\delta)}+\frac{\beta_{1}\left(\phi+\phi_{s}\right)}{(1+\phi)}\right) S_{2}}{\left(S_{1}+S_{2}\right)} \\
\frac{-\left(\frac{\alpha_{2}\left(\delta+\delta_{s}\right)}{(1+\delta)}+\frac{\beta_{2}\left(\phi+\phi_{s}\right)}{(1+\phi)}\right) S_{1}}{\left(S_{1}+S_{2}\right)} & \frac{\left(S_{1}+S_{2}\right)-\left(\frac{\alpha_{2}\left(\delta+\delta_{s}\right)}{(1+\delta)}+\frac{\beta_{2}\left(\phi+\phi_{s}\right)}{(1+\phi)}\right) S_{2}}{\left(S_{1}+S_{2}\right)}
\end{array}\right] \\
& \times\left[\begin{array}{ll}
P_{11} & P_{12} \\
P_{21} & P_{22}
\end{array}\right]\left[\begin{array}{l}
C_{1} \\
C_{2}
\end{array}\right] \text {. }
\end{aligned}
$$

In the representation above, the expansions for the matrices, $\mathcal{B}, \mathcal{P}$, and the elements $C_{1}, C_{2}$, are described in Equation A.4. Our final dynamic equations, $\dot{S}_{1}$ and $\dot{S}_{2}$, can now be written as the following system of dynamic equations

$$
\mathcal{H}\left(S_{1}, S_{2} ; \Omega\right),\left[\begin{array}{c}
\dot{S}_{1} \\
\dot{S}_{2}
\end{array}\right]=\mathcal{D}\left(S_{1}, S_{2} ; \boldsymbol{\Omega}\right)
$$

where $\Omega$ is the vector of all structural parameters of the model.

If $\mathcal{H}\left(S_{1}, S_{2} ; \boldsymbol{\Omega}\right)$ is invertible, then we can further reduce this to the following system 
of ordinary differential equations (ODEs):

$$
\begin{gathered}
{\left[\begin{array}{c}
\dot{S}_{1} \\
\dot{S}_{2}
\end{array}\right]=\left(\mathcal{H}\left(S_{1}, S_{2} ; \Omega\right)\right)^{(-1)} \mathcal{D}\left(S_{1}, S_{2} ; \Omega\right)} \\
{\left[\begin{array}{c}
\dot{S}_{1} \\
\dot{S}_{2}
\end{array}\right]=\mathcal{F}\left(S_{1}, S_{2} ; \Omega\right)=\left[\begin{array}{c}
\mathcal{F}_{1}\left(S_{1}, S_{2} ; \Omega\right) \\
\mathcal{F}_{2}\left(S_{1}, S_{2} ; \Omega\right)
\end{array}\right] .}
\end{gathered}
$$

Appendix B. Parameters.

Table B.1

Parameterizations $(\boldsymbol{\Omega})$

\begin{tabular}{|c|c|c|c|}
\hline Production Function & & Entry/Exit & \\
\hline$\alpha_{1}$ & 0.6 & $\gamma_{1}$ & 0.2 \\
$\beta_{1}$ & 0.2 & $\gamma_{2}$ & 0.1 \\
$\theta_{1}$ & 0.2 & $F_{1}$ & $\mathrm{BIF}$ \\
$\alpha_{2}$ & 0.2 & $F_{2}$ & 2 \\
$\beta_{2}$ & 0.6 & & \\
$\theta_{2}$ & 0.2 & & \\
\hline Government & & Elasticities & \\
\hline$D$ & 0.8 & $\eta_{11}$ & 2 \\
$\hat{D}^{s}$ & 0 & $\eta_{12}$ & 1 \\
$T^{s}$ & 0.25 & $\eta_{21}$ & 2 \\
$\hat{T}^{s}$ & 0 & $\eta_{22}$ & 2 \\
$\zeta_{1}$ & 0.2 & $\eta_{1 s}$ & 1 \\
$\zeta_{2}$ & 0.2 & $\eta_{2 s}$ & 1 \\
$\zeta_{l}$ & 0.4 & $\delta$ & 1 \\
$\zeta_{k}$ & 0.2 & $\delta_{s}$ & -1 \\
& & $\phi$ & 1 \\
& & $\phi_{s}$ & -1 \\
\hline
\end{tabular}




\section{References}

Barnett, W. A., 2000. Perspective on the current state of macroeconomic theory. In: W.A.Barnett, Serletis, A. (Eds.), The theory of monetary aggregation. North Holland, pp. 600-601.

Barnett, W. A., Duzhak, E., 2008. Non-robust dynamic inferences from macroeconometric models: Bifurcation stratification of confidence regions. Physica A 387, 3817-3825.

Barnett, W. A., Duzhak, E., 2010. Empirical assessment of bifurcation regions within new keynesian models. Economic Theory 45, 99-128.

Barnett, W. A., He, Y., 2002. Stabilization policy as bifurcation selection: would stabilization policy work if the economy really were unstable? Macroeconomic Dynamics 6 (05), 713-747.

Barnett, W. A., He, Y., 2004. Bifurcations in macroeconomic models. In: S. Dowrick, R. Pitchford, S. T. (Eds.), Economic growth and macroeconomic dynamics: Recent developments in economic theory. Cambridge University Press, pp. 95-112.

Barnett, W. A., He, Y., 2006. Singularity bifurcations. Journal of Macroeconomics $28(1), 5-22$.

Barnett, W. A., He, Y., (2010). Existence of singularity bifurcation in an Eulerequations model of the United States economy: Grandmont was right. Economic Modelling 27 (6), 1345-1354.

Benhabib, J., Day, R. H., 1982. A characterization of erratic dynamics in, the overlapping generations model. Journal of Economic Dynamics and Control 4, 37 55.

Benhabib, J., Nishimura, K., 1979. The hopf bifurcation and the existence and stability of closed orbits in multisector models of optimal economic growth. Journal of Economic Theory 21 (3), $421-444$.

Bergstrom, A. R., Nowman, K. B., Wymer, C. R., 1992. Gaussian estimation of a second order continuous time macroeconometric model of the UK. Economic Modelling 9 (4), 313 - 351.

Boldrin, M., Woodford, M., 1990. Equilibrium models displaying endogenous fluctuations and chaos : A survey. Journal of Monetary Economics 25 (2), $189-222$.

Grandmont, J.-M., 1985. On endogenous competitive business cycles. Econometrica $53(5), 995-1045$.

Kuznetsov, Y. A., 2004. Elements of applied bifurcation theory, 3rd Edition. Springer.

Leeper, E., Sims, C., 1994. Toward a modern macro model usable for policy analysis. NBER Macroeconomic Annual, 81-117.

Veloce, W., Zellner, A., 1985. Entry and empirical demand and supply analysis for competitive industries. Journal of Econometrics 30 (1-2), 459-471.

Zellner, A., Israilevich, G., 2005. Marshallian macroeconomic model: A progress report. Macroeconomic Dynamics 9 (02), 220-243. 